\title{
Proportional-integral-plus (PIP) control of time delay systems
}

\author{
C J Taylor, A Chotai and P C Young
}

Centre for Research on Environmental Systems and Statistics (CRES), Lancaster University

\begin{abstract}
The paper shows that the digital proportional-integral-plus (PIP) controller formulated within the context of non-minimum state space (NMSS) control system design methodology is directly equivalent, under certain non-restrictive pole assignment conditions, to the equivalent digital Smith predictor (SP) control system for time delay systems. This allows SP controllers to be considered within the context of NMSS state variable feedback control, so that optimal design methods can be exploited to enhance the performance of the SP controller. Alternatively, since the PIP design strategy provides a more flexible approach, which subsumes the SP controller as one option, it provides a superior basis for general control system design. The paper also discusses the robustness and disturbance response characteristics of the two PIP control structures that emerge from the analysis and demonstrates the efficacy of the design methods through simulation examples and the design of a climate control system for a large horticultural glasshouse system.
\end{abstract}

Keywords: Smith predictor, proportional-integral-plus (PIP), time delay systems, pole assignment, robustness

NOTATION
$A\left(z^{-1}\right)$
$B\left(z^{-1}\right)$
$\hat{A}\left(z^{-1}\right), \hat{B}\left(z^{-1}\right)$
$\boldsymbol{d}_{\mathrm{PIP}}, \boldsymbol{d}_{\mathrm{SP}}$
$D\left(z^{-1}\right)$
$\mathbf{F}$
$F\left(z^{-1}\right)$
$F_{\mathrm{S}}\left(z^{-1}\right)$
$\boldsymbol{g}$
$G\left(z^{-1}\right)$
$G_{\mathrm{S}}\left(z^{-1}\right)$
$\boldsymbol{k}$
$\boldsymbol{k}_{\mathrm{PIP}}, \boldsymbol{k}_{\mathrm{SP}}$
$k_{\mathrm{I}}$
$k_{\mathrm{S}}$
$\boldsymbol{h}$
$m$

The MS was received on 14 March 1997 and was accepted for publication on 29 August 1997.

$n$

denominator polynomial of system transfer function

numerator polynomial of system transfer function

estimated polynomials

vectors of PIP and SP-PIP desired

closed-loop coefficients

desired closed-loop characteristic

polynomial

state transition matrix

PIP control feedback polynomial

SP-PIP control feedback polynomial

state space input vector

PIP control feedforward polynomial

SP-PIP control feedforward polynomial

control gain vector

PIP and SP-PIP control gain vectors

$\mathrm{PIP}$ integral gain

SP-PIP integral gain

state space observation vector

order of model numerator polynomial

I01097 @ IMechE 1998

$\begin{array}{ll}\boldsymbol{p}_{\mathrm{PIP}}, \boldsymbol{p}_{\mathrm{SP}} & \text { PIP and SP-PIP open-loop charac- } \\ \mathbf{P} & \text { teristic polynomial coefficients } \\ & \text { estimated parameter covariance } \\ & \text { matrix } \\ u(k) & \text { control input } \\ v(k) & \text { load disturbance } \\ \boldsymbol{w} & \text { state space command input vector } \\ \boldsymbol{x}(k) & \text { state vector } \\ y(k) & \text { measured output } \\ y_{\mathrm{d}}(k) & \text { command (desired) input } \\ z(k) & \text { integral of error state } \\ z^{-i} & \text { backward shift operator, } z^{-i} y(k)= \\ & y(k-i) \\ \nabla & \text { difference operator }=1-z^{-1} \\ \boldsymbol{\Sigma}_{\mathrm{PIP}}, \boldsymbol{\Sigma}_{\mathrm{SP}} & \text { PIP and SP-PIP pole assignment } \\ \tau & \text { matrices } \\ \tau & \text { time delay }\end{array}$

\section{INTRODUCTION}

Pure time delay or 'dead-time' is a common feature of many physical systems and is a major concern in control system design. When the time delay is short in comparison with the dominant time constants of the system it can be handled fairly easily. For instance, in the case of order of model denominator polynomial teristic polynomial coefficients matrix state space command input vector command (desired) input backward shift operator, $z^{-i} y(k)=$ difference operator $=1-z^{-1}$ matrices me delay 
discrete-time, sampled data control systems, where the time delay can be approximated by an integral number of sampling intervals, transfer function or state space design methods are able to easily absorb the time delay into the system model. In the situation where the time delay is much larger than the dominant time constants of the system, however, it represents an important challenge since the enlarged model will result in a very high order control system design.

Over many years, the Smith predictor (SP) has proven to be one of the most popular approaches to the design of controllers for time delay systems. Although initially formulated for continuous time systems (1), the SP can be implemented in any digital control scheme, such as conventional digital PI/PID (proportional-integral/ proportional-integral-derivative) controllers or the more sophisticated proportional-integral-plus (PIP) controllers proposed in recent years [e.g. see references (2) to (4)]. The present paper goes further, however, and demonstrates how, under certain non-restrictive pole assignment conditions, the PIP controller is exactly equivalent to the digital SP controller but has much greater inherent flexibility and robustness in design terms. In particular, the inherent state space formulation of the PIP controller allows for the application of all methods of state space control optimal design. In addition, optimal selection of the weighting matrices in the optimal criterion function can allow for the achievement of multiple objectives (5).

\section{PROPORTIONAL-INTEGRAL-PLUS (PIP) CONTROL}

A number of previous papers have been concerned with the true digital control (TDC) design philosophy and its practical embodiment in the non-minimum state space (NMSS) approach to control system design [e.g. see references (2) to (4)]. Although the approach can be applied directly to multivariable systems described by backward shift (3), continuous-time or delta (5) operator models, for the purposes of the present paper the discussion is restricted to the standard, backward shift operator, transfer function representation of an $n$th order, linear, single-input, single-output system, i.e.

$$
\begin{aligned}
y(k) & =\frac{\left(b_{\tau} z^{-1}+\cdots+b_{\tau+m-1} z^{-m}\right) z^{-\tau+1}}{1+a_{1} z^{-1}+\cdots+a_{n} z^{-n}} u(k) \\
& =\frac{B\left(z^{-1}\right) z^{-\tau+1}}{A\left(z^{-1}\right)} u(k)
\end{aligned}
$$

where $\tau>0$ is the pure time (transport) delay in sampling intervals of $\Delta t$ time units. It is easy to show that the model (1) can be represented by the following NMSS equations:

$$
\begin{aligned}
& \boldsymbol{x}(k)=\mathbf{F} \boldsymbol{x}(k)+\boldsymbol{g} u(k-1)+\boldsymbol{w} y_{\mathrm{d}}(k) \\
& y(k)=\boldsymbol{h} \boldsymbol{x}(k)
\end{aligned}
$$

where the $n+m+\tau-1$ dimensional NMSS state vector $\boldsymbol{x}(k)$ is defined as follows:

$$
\begin{aligned}
& \boldsymbol{x}(k)=\left[\begin{array}{lllll}
y(k) & y(k-1) & \cdots & y(k-n+1) & u(k-1)
\end{array}\right. \\
& u(k-2) \cdots u(k-m-\tau+2) z(k)]^{\mathrm{T}}
\end{aligned}
$$

in which $z(k)$ is the integral of error between the reference or command input $y_{\mathrm{d}}(k)$ and the sampled output $y(k)$, i.e.

$$
z(k)=z(k-1)+\left[y_{\mathrm{d}}(k)-y(k)\right]
$$

When $\tau>1$, the state matrices are defined as follows [for brevity, the case when $\tau=1$ is not shown, but is straightforward to obtain; see reference (2)]:

$$
\begin{aligned}
& \mathbf{F}=\left[\begin{array}{ccccccccccccccc}
-a_{1} & -a_{2} & \cdots & -a_{n-1} & -a_{n} & 0 & 0 & \cdots & 0 & b_{\tau} & b_{\tau+1} & \cdots & b_{\tau+m-2} & b_{\tau+m-1} & 0 \\
1 & 0 & \cdots & 0 & 0 & 0 & 0 & \cdots & 0 & 0 & 0 & \cdots & 0 & 0 & 0 \\
0 & 1 & \cdots & 0 & 0 & 0 & 0 & \cdots & 0 & 0 & 0 & \cdots & 0 & 0 & 0 \\
\vdots & \vdots & \ddots & \vdots & \vdots & \vdots & \vdots & \ddots & \vdots & \vdots & \vdots & \ddots & \vdots & \vdots & \vdots \\
0 & 0 & \cdots & 1 & 0 & 0 & 0 & \cdots & 0 & 0 & 0 & \cdots & 0 & 0 & 0 \\
0 & 0 & \cdots & 0 & 0 & 0 & 0 & \cdots & 0 & 0 & 0 & \cdots & 0 & 0 & 0 \\
0 & 0 & \cdots & 0 & 0 & 1 & 0 & \cdots & 0 & 0 & 0 & \cdots & 0 & 0 & 0 \\
0 & 0 & \cdots & 0 & 0 & 0 & 1 & \cdots & 0 & 0 & 0 & \cdots & 0 & 0 & 0 \\
\vdots & \vdots & \ddots & \vdots & \vdots & \vdots & \vdots & & \vdots & \vdots & \vdots & & \vdots & \vdots & \vdots \\
0 & 0 & \cdots & 0 & 0 & 0 & 0 & \cdots & 0 & 0 & 0 & \cdots & 1 & 0 & 0 \\
a_{1} & a_{2} & \cdots & a_{n-1} & a_{n} & 0 & 0 & \cdots & 0 & -b_{\tau} & -b_{\tau+1} & \cdots & -b_{\tau+m-2} & -b_{\tau+m-1} & 1
\end{array}\right] \\
& \boldsymbol{g}=\left[\begin{array}{lllllllllll}
0 & 0 & \cdots & 0 & 1 & 0 & 0 & \cdots & 0 & 0 & 0
\end{array}\right]^{\mathrm{T}} \\
& \boldsymbol{d}=\left[\begin{array}{lllllllllll}
0 & 0 & \cdots & 0 & 0 & 0 & 0 & \cdots & 0 & 0 & 1
\end{array}\right]^{\mathrm{T}} \\
& \boldsymbol{h}=\left[\begin{array}{lllllllllll}
1 & 0 & \cdots & 0 & 0 & 0 & 0 & \cdots & 0 & 0 & 0
\end{array}\right]
\end{aligned}
$$


The proportional-integral-plus (PIP) controller is simply the state variable feedback (SVF) control law associated with this NMSS model, i.e.

$$
u(k)=-\boldsymbol{k}^{\mathrm{T}} \boldsymbol{x}(k)
$$

where $\boldsymbol{k}$ is the $(n+m+\tau-1)$-dimensional SVF control gain vector, i.e.

$$
\boldsymbol{k}=\left[\begin{array}{lllllllll}
f_{0} & f_{1} & \cdots & f_{n-1} & g_{1} & g_{2} & \cdots & g_{m+\tau-2} & -k_{\mathrm{I}}
\end{array}\right]^{\mathrm{T}}
$$

Since all the state variables in $\boldsymbol{x}(k)$ are readily stored in the digital computer, the PIP controller (6) is easy to implement in practice. Moreover, the inherent SVF formulation allows for the exploitation of any SVF design procedure: e.g. optimization in terms of linear quadratic (LQ), linear quadratic Gaussian (LQG), $H_{\infty}$, or other cost functions such as the linear exponential of quadratic Gaussian (LEQG). However, the present paper concentrates initially on the case of pole assignment, where the algebraic results are most transparent. In this context, the desired closed-loop characteristic polynomial is defined as follows:

$$
D\left(z^{-1}\right)=1+d_{1} z^{-1}+\cdots+d_{n+m+\tau-1} z^{-(n+m+\tau-1)}
$$

where, as usual in pole assignment design, the coefficients $d_{1}, d_{2}, \ldots, d_{n+m+\tau-1}$ are selected to ensure that the closed-loop poles are at positions in the complex $z$ plane, which provide satisfactory closed-loop performance.

\section{THE IMPORTANCE OF STRUCTURE IN PIP CONTROL DESIGN}

The PIP controller is so called because, in the more conventional block diagram terms shown in Fig. 1, it can be considered as one particular extension of the PI controller, in which the PI action is enhanced by higherorder forward path and feedback compensators $1 / G\left(z^{-1}\right)$ and $F_{1}\left(z^{-1}\right)$ respectively, where

$$
\begin{aligned}
F_{1}\left(z^{-1}\right) & =f_{1} z^{-1}+f_{2} z^{-2}+\cdots+f_{n-1} z^{-(n-1)} \\
G\left(z^{-1}\right) & =1+g_{1} z^{-1}+\cdots+g_{m+\tau-2} z^{-(m+\tau-2)}
\end{aligned}
$$

Defining $F\left(z^{-1}\right)=f_{0}+F_{1}\left(z^{-1}\right)$, the closed-loop transfer function of the PIP controlled system takes the following general form:

$$
\begin{gathered}
y(k)=\frac{k_{\mathrm{I}} B\left(z^{-1}\right) z^{-\tau+1}}{\nabla\left[G\left(z^{-1}\right) A\left(z^{-1}\right)+F\left(z^{-1}\right) B\left(z^{-1}\right) z^{-\tau+1}\right]} y_{\mathrm{d}}(k) \\
+k_{\mathrm{I}} B\left(z^{-1}\right) z^{-\tau+1}
\end{gathered}
$$

where $\nabla=1-z^{-1}$ is the difference operator and $k_{\mathrm{I}}$ is the integral of error gain.

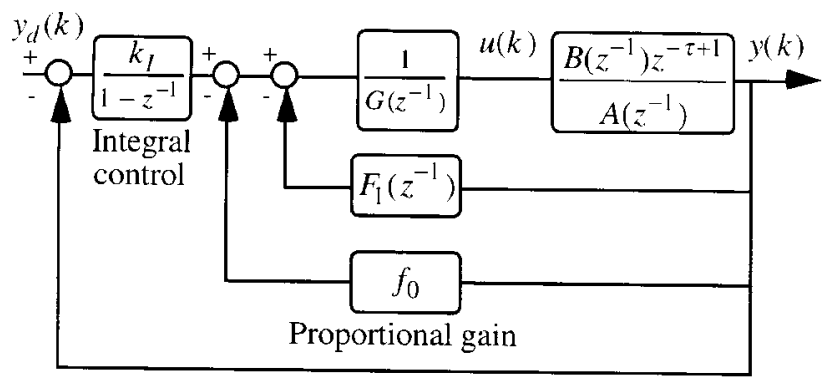

Fig. 1 PIP control with feedback structure

It is straightforward to eliminate the inner loop of Fig. 1 to form a single forward path (or pre-compensation) transfer function form of the controller $(6)$. One way of representing the forward path controller is illustrated in Fig. 2a: this shows how the model (with the circumflexes denoting the estimated parameter polynomials and estimated time delay) acts as a source of information on the state variables, while the measured output is also fed back to ensure that, at steady state, the desired command level is maintained despite any disturbance inputs. The forward path controller may also be arranged into an equivalent internal model structure [e.g. see reference (7)], with a feedback of the model mismatch, as illustrated in Fig. $2 b$.

\subsection{Closed-loop behaviour}

Naturally, the closed-loop forms of Fig. 2 are only identical to those given by equation (10) for the case of zero model mismatch. The choice of structure, therefore, has important consequences, both for the robustness of the final design to parametric uncertainty and for the disturbance rejection characteristics. In particular, the results discussed later, together with practical experience of these systems, has suggested that the feedback form illustrated in Fig. 1 is generally more robust to uncertainty in the estimated system dynamics. As usual and not surprisingly, the internal model structure, which is inherent in the forward path form, yields a controller that is relatively sensitive to uncertainty in the estimate of $A\left(z^{-1}\right)$, especially for marginally stable or unstable systems (6).

By contrast, in the case of zero mismatch, the unity feedback aspect of the forward path form offers disturbance rejection characteristics that are usually superior to those of the feedback form, since they are similar in dynamic terms to those associated with the designed command response. This is clear if the closed-loop transfer functions are considered for the feedback $y_{\mathrm{FB}}(k)$ and forward path $y_{\mathrm{FP}}(k)$ structures in the case of a load disturbance $v(k)$, i.e. 


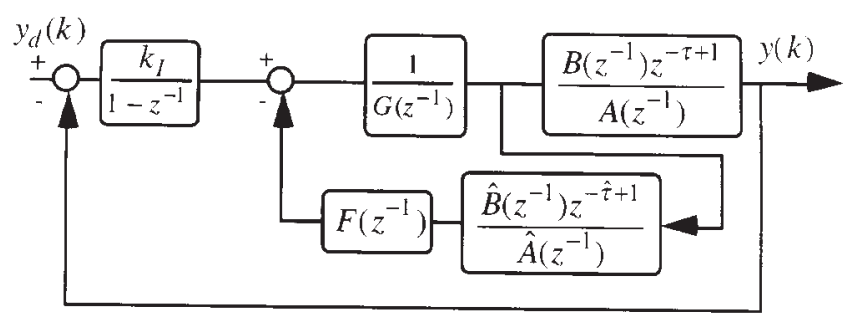

(a)

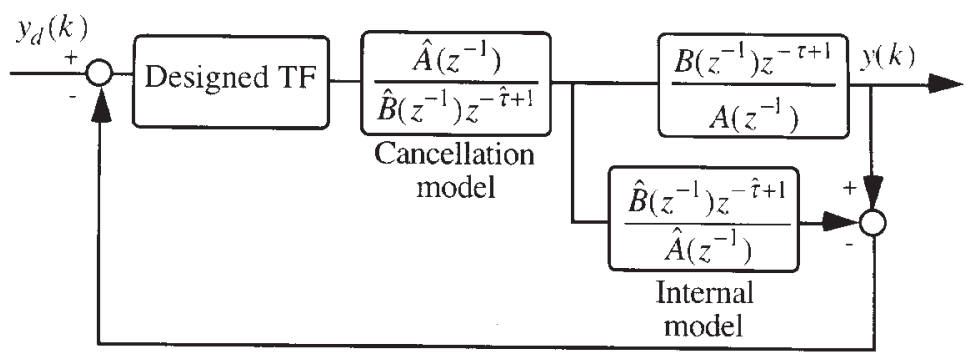

(b)

$$
\begin{aligned}
& \text { Designed TF: } \\
& \qquad \frac{k_{\mathrm{I}} \hat{B}\left(z^{-1}\right) z^{-\hat{\imath}+1}}{\nabla\left[G\left(z^{-1}\right) \hat{A}\left(z^{-1}\right)+F\left(z^{-1}\right) \hat{B}\left(z^{-1}\right) z^{-\hat{\imath}+1}\right]+k_{\mathrm{I}} \hat{B}\left(z^{-1}\right) z^{-\hat{\imath}+1}}
\end{aligned}
$$

Fig. 2 Two identical structures for forward path PIP control

$$
\begin{aligned}
& y_{\mathrm{FB}}(k)=\frac{\nabla G\left(z^{-1}\right) A\left(z^{-1}\right)}{\nabla\left[G\left(z^{-1}\right) A\left(z^{-1}\right)+F\left(z^{-1}\right) B\left(z^{-1}\right) z^{-\tau+1}\right)+k_{\mathrm{I}} B\left(z^{-1}\right) z^{-\tau+1}} v(k) \\
& y_{\mathrm{FP}}(k)=\frac{\nabla\left[G\left(z^{-1}\right) A\left(z^{-1}\right)+F\left(z^{-1}\right) B\left(z^{-1}\right) z^{-\tau+1}\right]}{\nabla\left[G\left(z^{-1}\right) A\left(z^{-1}\right)+F\left(z^{-1}\right) B\left(z^{-1}\right) z^{-\tau+1}\right]+k_{\mathrm{I}} B\left(z^{-1}\right) z^{-\tau+1}} v(k)
\end{aligned}
$$

Straightforward algebraic manipulation of equation (12) shows that

$$
y_{\mathrm{FP}}(k)=\left\{1-\frac{k_{\mathrm{I}} B\left(z^{-1}\right) z^{-\tau+1}}{\nabla\left[G\left(z^{-1}\right) A\left(z^{-1}\right)+F\left(z^{-1}\right) B\left(z^{-1}\right) z^{-\tau+1}\right]+k_{\mathrm{I}} B\left(z^{-1}\right) z^{-\tau+1}}\right\} v(k)
$$

whereas such a simple result is not possible in the case of equation (11). In other words, the disturbance response in the forward path case is equal to one minus the designed command response, thus ensuring similar disturbance response dynamics to those specified by the designer in relation to the command input response.

As will be seen later, another very desirable characteristic of the forward path structure is that, in general, the actuator signal is much smoother than that produced by the feedback form of the controller. This has important practical implications for reducing actuator wear $(3,6)$. In the case of the feedback structure the situation is more complex, however, since past, noisy values of the disturbed output are involved in the control signal synthesis because of the feedback filter $F\left(z^{-1}\right)$. In practice, this may result in a less desirable disturbance response to that obtained with the forward path form, as illustrated in Section 7 below. Although not considered in the present paper, the disturbance response characteristics of the two structures can also be compared in standard (Bode/Nyquist) frequency domain terms $(\mathbf{8})$.

\section{THE SP-PIP CONTROLLER FOR TIME DELAY SYSTEMS}

The PIP controller automatically handles a pure time delay by simply feeding back sufficient past values of the input variable to span the time delay. However, in the case of systems with a long time delay, where the choice of a coarser sampling interval is not possible as a means of reducing the dimension of the NMSS, this may require an excessive definition of the NMSS order and, consequently, an unacceptably large number of gains in the $G\left(z^{-1}\right)$ filter. In this situation, it seems more efficient and parsimonious to employ an SP-type controller in the standard manner to deal with the time delay. In the resulting SP-PIP controller (9), the delay is external to the control loop when there is no model mismatch, as shown in Fig. 3, and so the specification of system performance is obtained by defining an $(n+m)$-dimensional non-minimal state vector, based on the unit delay model [i.e. equation (1) with $\tau=1]$. 


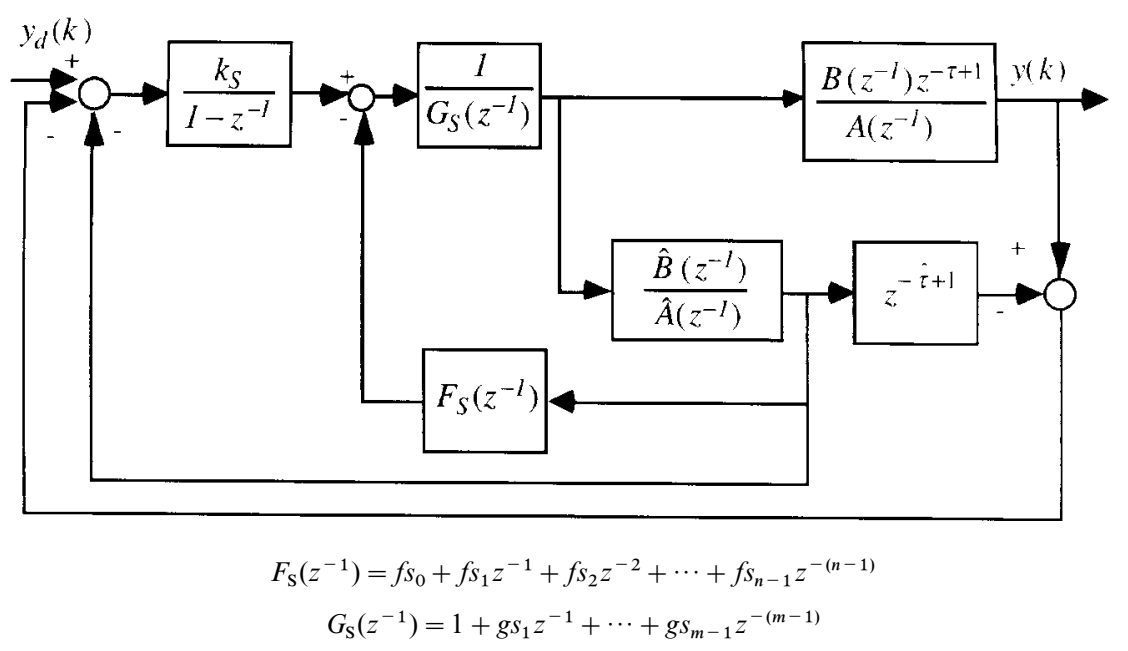

Fig. 3 PIP control with the Smith predictor (the SP-PIP system)

The closed-loop transfer function in the SP-PIP case reduces to the standard PIP closed-loop (10), but with $B\left(z^{-1}\right) z^{-\tau+1}$ replaced by just $B\left(z^{-1}\right)$ in the denominator, i.e.

$$
\begin{aligned}
& y(k)= \\
& \quad \frac{k_{\mathrm{S}} B\left(z^{-1}\right) z^{-\tau+1}}{\nabla\left[G_{\mathrm{S}}\left(z^{-1}\right) A\left(z^{-1}\right)+F_{\mathrm{S}}\left(z^{-1}\right) B\left(z^{-1}\right)\right]+k_{\mathrm{S}} B\left(z^{-1}\right)} y_{\mathrm{d}}(k)
\end{aligned}
$$

where $F_{\mathrm{S}}\left(z^{-1}\right), G_{\mathrm{S}}\left(z^{-1}\right)$ and $k_{\mathrm{S}}$ are the PIP filters designed in this manner. Note that here the SP-PIP and standard PIP control gain vectors have been calculated from models with different numerator orders, so that if $\tau>1$ the order of the $G\left(z^{-1}\right)$ filter will be higher than $G_{\mathrm{S}}\left(z^{-1}\right)$.

\section{RELATIONSHIP BETWEEN THE PIP AND SP-PIP CONTROL GAINS}

The characteristic polynomial of the SP-PIP system is of the order $n+m$, compared with $n+m+\tau-1$ in the standard PIP case. However, equivalence between the two may be obtained by the following theorem. control system are constrained to be the same as those of the SP-PIP case.

Moreover, under these simple conditions, the standard PIP gain vector $\boldsymbol{k}_{\mathrm{PIP}}$ is given by

$$
\boldsymbol{k}_{\mathrm{PIP}}=\boldsymbol{\Sigma}_{\mathrm{PIP}}^{-1} \cdot \boldsymbol{\Sigma}_{\mathrm{SP}} \cdot \boldsymbol{k}_{\mathrm{SP}}
$$

where $\boldsymbol{\Sigma}_{\text {PIP }}$, which is defined in the Appendix, is the parameter matrix employed for the design of a pole assignment controller for the standard PIP control system (2), $\boldsymbol{\Sigma}_{\mathrm{SP}}$ is the parameter matrix employed for the design of a pole assignment controller for the SP-PIP control system (defined in a similar manner to $\boldsymbol{\Sigma}_{\text {PIP }}$ but based on the unit delay model) and $\boldsymbol{k}_{\mathrm{SP}}$ is the SP-PIP gain vector. The proof of this theorem is given in the Appendix.

\section{THE COMPLETE EQUIVALENCE OF PIP AND SP-PIP CONTROLLERS}

To consider the more general case, when there is model mismatch, the SP-PIP controller is simply converted into a unity feedback, forward path pre-compensation implementation. In this case, the control law (which remains identical to that illustrated in Fig. 3) takes the form

$$
u(k)=\frac{k_{\mathrm{S}} \hat{A}\left(z^{-1}\right)}{\nabla\left[G_{\mathrm{S}}\left(z^{-1}\right) \hat{A}\left(z^{-1}\right)+F_{\mathrm{S}}\left(z^{-1}\right) \hat{B}\left(z^{-1}\right)\right]+k_{\mathrm{S}}\left[\hat{B}\left(z^{-1}\right)-\hat{B}\left(z^{-1}\right) z^{-\hat{\imath}+1}\right]}\left[y_{\mathrm{d}}(k)-y(k)\right]
$$

Theorem 1 has already established that, with appropriate selection of poles, the SP-PIP closed-loop transfer function must be identical to the closed-loop transfer function resulting from standard PIP control in the case of a perfectly known model. Clearly, for this result to hold when both controllers are implemented in a forward path form [see Fig. 2 for PIP, equation (16) for SP-PIP], then the control filter in each case must be identical. Moreover, this result applies even in the case of mismatch and disturbance inputs, since the control filter is invariant to such effects. In other words, the PIP controller implemented
1. The $(\tau-1)$ poles in the standard PIP control system are assigned to the origin of the complex $z$ plane.

2. The remaining $(n+m)$ poles of the standard PIP 
with the forward path structure is always completely identical to the SP-PIP controller. However, it is important to stress that this is not the case for the standard feedback structure of the PIP controller.

\section{EXAMPLES}

In order to illustrate clearly the results obtained in previous sections, it is advantageous to consider first the following marginally stable, non-minimum phase model, which has a total of five samples of pure time delay $(\tau=5)$ :

$$
y(k)=\frac{-z^{-5}+2 z^{-6}}{1-1.7 z^{-1}+z^{-2}} u(k)
$$

When the four closed-loop poles for the SP-PIP control system are all assigned to 0.5 , while the additional four poles in the standard PIP case are set to zero, as discussed in Section 5, the closed-loop transfer function is identical for all three cases of Figs 1, 2 and 3. However, in the presence of model mismatch or disturbance inputs to the system, the SP-PIP and feedback PIP closed-loop transfer functions are different, while, as expected, the PIP controller implemented in the forward path form remains identical to the SP-PIP case.

For example, Fig. 4 illustrates the response of the two formulations to a load disturbance, where, as mentioned in Section 3 and following from equation (13), the forward path form of the controller (i.e. Fig. 2 or Fig. 3) results in a smoother response than the feedback form
(Fig. 1), thus requiring much reduced actuator movement and resulting in less actuator wear.

\subsection{Robustness to parametric uncertainty}

An important practical consideration in model-based control system design is the robustness of the control system performance to uncertainty associated with the model parameters. This problem can be handled in many ways but, with the current wide availability of powerful desktop computers, Monte Carlo (MC) analysis provides one of the simplest and most attractive approaches to the problem. The MC analysis employed in the present paper is based on the parameter covariance matrix $\mathbf{P}$ generated by the parameter estimation algorithm used for data-based modelling. In other words, the model parameters for each realization in the MC analysis are selected randomly from the joint probability distribution defined by the $\mathbf{P}$ matrix and the sensitivity of the PIP controlled system to parametric uncertainty is then evaluated from the ensemble of resulting closed-loop response characteristics (e.g. time domain, frequency domain, pole-zero positions, etc.).

In the present context, this kind of MC analysis is useful for evaluating the performance differences between the PIP and SP-PIP control structures. Zero mean, white noise, with unity variance, is added to the output of the system (17) to provide a noise-signal ratio of 0.2 and the refined instrumental variable (RIV) identification algorithm (10) is used to obtain estimates
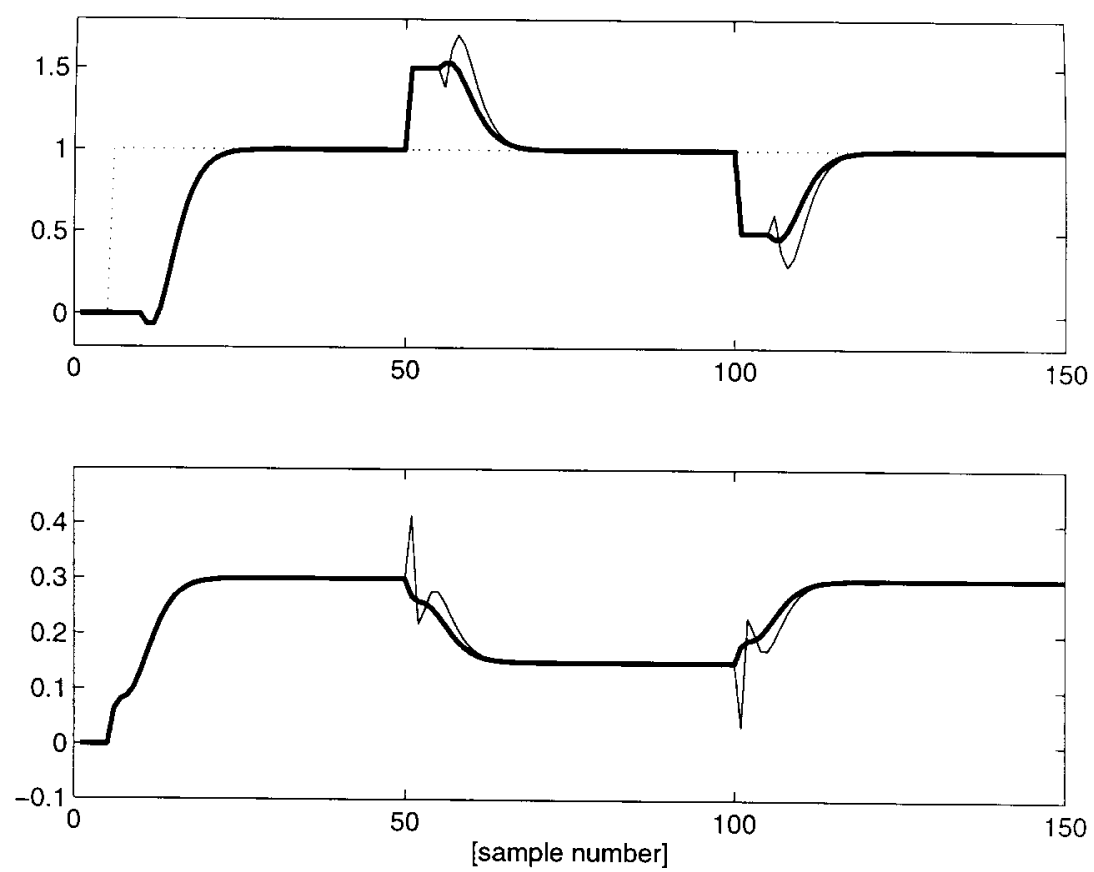

Fig. 4 Comparison of feedback PIP (thin trace) and SP-PIP (thick trace) control of equation (17); both the system output (top) and control input (bottom) are shown. A load disturbance of magnitude 0.5 is added to the output from the fiftieth to the hundredth samples 
of the model parameters and their associated covariance matrix $\mathbf{P}$. In order to better illustrate the effects of uncertainty, however, $\mathbf{P}$ is artificially inflated by a factor of 4 before being employed in the MC studies.

Figure 5 compares the resulting $\mathrm{MC}$ responses of the various PIP controllers discussed above to a step change in the command input. Clearly, in this case, the cancellation inherent in the forward path structure (whether implemented in the form of either Fig. 2 or Fig. 3) has made it more sensitive to the parametric uncertainty. Note that although the forward path time responses in Fig. 5 appear relatively well behaved, slowly growing oscillations can be seen in the Monte Carlo envelope and these become more apparent if the time axis is extended further. In fact, an examination of the closed-loop poles reveals that 46 per cent of the SP-PIP responses are unstable, compared to none in the feedback PIP case.

In considering the results of Fig. 5, however, it should
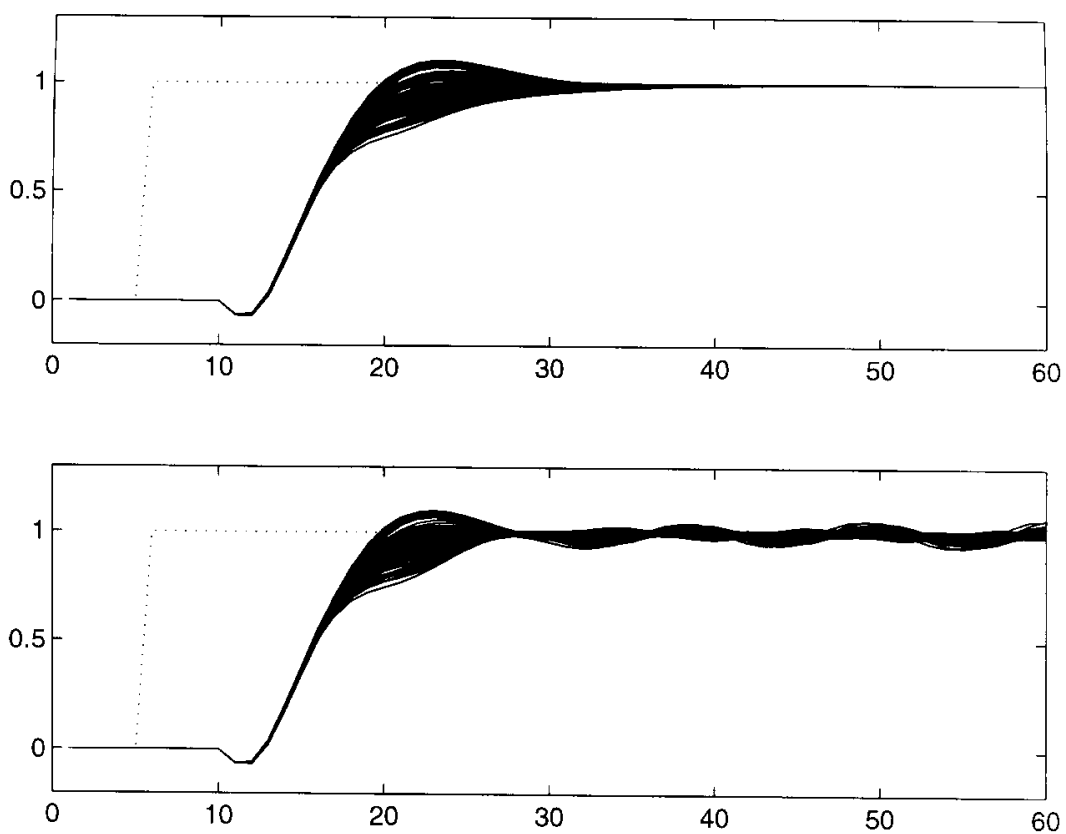

Fig. 5 Comparison of feedback PIP (top: 0 per cent unstable) and SP-PIP (bottom: 46 per cent unstable) control of the system (17), with 100 Monte Carlo realizations
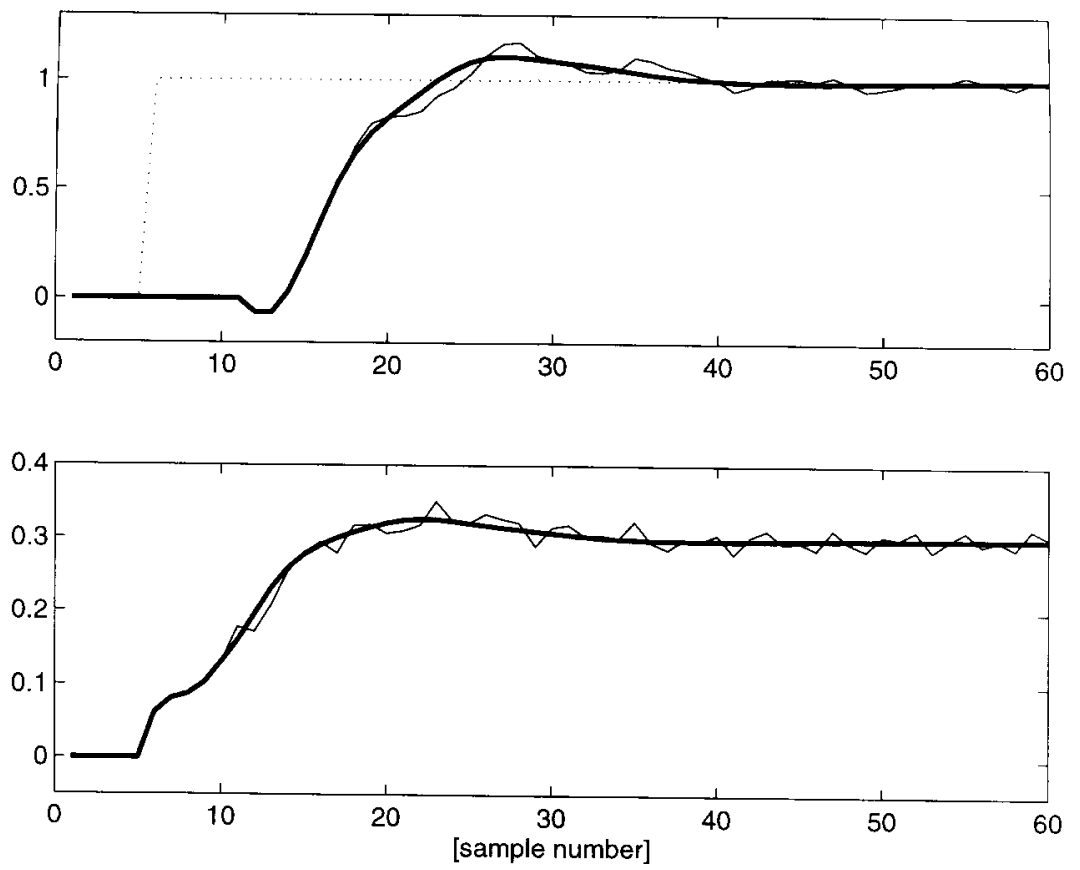

Fig. 6 Comparison of feedback PIP (thin) and SP-PIP (thick) control of the system (17), with mismatch in the time delay; both the system output (top) and control input (bottom) are shown 
be emphasized that not only is the system in this case quite difficult to control (with two open-loop poles on the unit circle in the complex $z$ plane) but the parametric uncertainty has been artificially enlarged to facilitate the comparison. In fact, both forms of PIP control are very robust to more realistic modelling uncertainties, as demonstrated in various practical examples [e.g. see references (3) and (8)].

\subsection{Robustness to time delay uncertainty}

One assumption in the MC analysis discussed above is that the model parameters are subject to uncertainty but the structure $(n, m, \tau)$ remains constant in all the realizations. Within the context of the present paper, however, it is interesting to examine the situation where this assumption is relaxed to allow for uncertainty in the time delay. In particular, the results in Fig. 6 are based on single simulations using the system (17) with the control- ler design based on the original five-sample delay, but with the simulated delay changed to $\tau=6$.

Typically, as in Fig. 6, the forward path controller performs better than the standard feedback structure. In this example, if the system time delay is increased still further so that $\tau=7$, then the feedback form of the controller is unstable, while the response of the alternative forward path structure remains similar to that illustrated in Fig. 6. In fact, the forward path controller does not yield an unstable response until the system delay is increased to greater than $\tau=16$ although, by this stage, the performance is severely degraded.

\subsection{Practical example: control of greenhouse microclimate}

Conventional greenhouse climate controllers are usually based on continuous-time PI or PID algorithms, manually tuned to achieve adequate, although rather poor, tracking
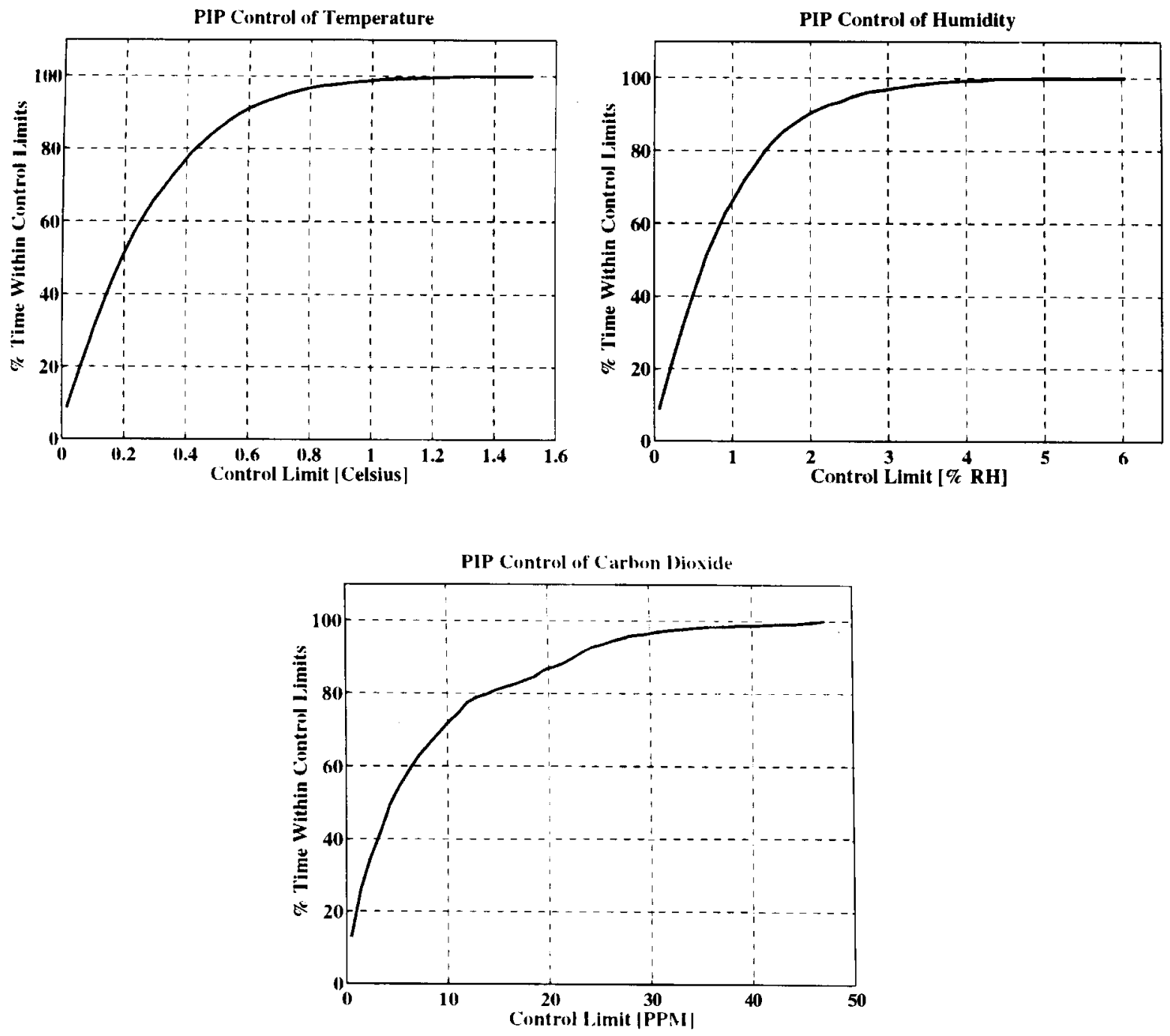

Fig. 7 Performance of PIP control: temperature, relative humidity and carbon dioxide. [Reprinted from reference $(\mathbf{8})$ ] 
of commands. Previous publications [e.g. references (2) and (8)] have shown how the model-based PIP methodology can achieve much tighter control of the climate variables allowing, for example, changing optimal set points to be followed without difficulty. Evaluation of PIP control on a large horticultural 'Venlo' greenhouse at Silsoe Research Institute (SRI) was carried out over a 3 month implementation period during the 1993-4 growing season with a tomato crop (8). Figure 7 shows the control performance of the climate variables over the entire evaluation period. Each plot shows the percentage of the validation period that a control variable was inside a certain control limit. For example, air temperature was less than $1{ }^{\circ} \mathrm{C}$ away from the set point for 98 per cent of the validation period and was never more than $1.5^{\circ} \mathrm{C}$ away from the desired temperature.

In the case of temperature control, the linear control model of the form (1), identified from experimental data collected in the greenhouse at SRI, is characterized by time delays of up to $30 \mathrm{~min}$. In addition, control system design is complicated by the fact that the internal climate of the greenhouse is affected to a large extent by external weather disturbances, particularly solar radiation. It is clear from the results of the Venlo experiments that, in the case of internal air temperature, the forward path form of PIP control and its equivalent SP-PIP implementation are superior to the standard feedback PIP controller. In particular, these forward path controllers yield a similar closed-loop temperature response, but there is a significant reduction in valve aperture movement, as illustrated in Fig. 8.

\section{CONCLUSIONS}

This paper has shown how the Smith predictor (SP) approach to the control of time delay systems can be
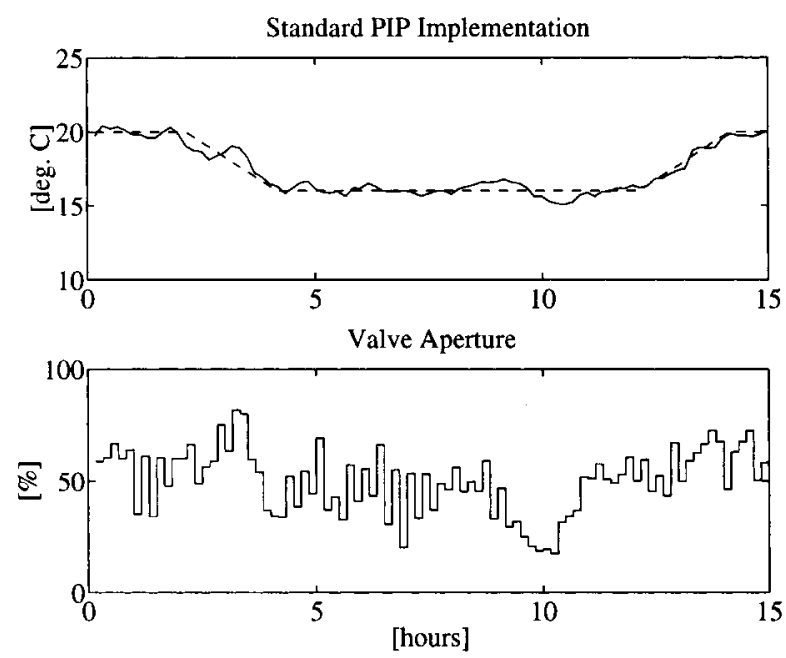

formulated within the context of non-minimum state space (NMSS) digital control system design. This can be achieved either explicitly, as an SP addition to the proportional-integral-plus (PIP) controller, or implicitly, in a unity feedback gain, forward path implementation of the PIP pole assignment controller. It is felt that this approach provides a more formal and satisfactory treatment of Smith predictor design than has been suggested heretofore, since it allows optimal state space design methods to be fully exploited in order to enhance the performance of the SP controller. However, since the PIP design strategy provides a more flexible and unified approach, which subsumes the SP controller as one option, it provides a superior basis for general control system design.

It is important to emphasize that PIP pole assignment design of this SP type, in which the closed-loop poles associated with time delay are assigned to the origin of the complex $z$ plane, is not necessarily the most robust solution to the control design problem. In particular, the alternative LQ optimal PIP control system design, which effectively subsumes the SP approach in the time delay situation, will normally yield better and more robust closed-loop behaviour, making it more desirable for practical applications. The advantage of the PIP approach (without an explicit SP) is that full order pole assignment or LQ control can be handled in either the feedback or the forward path form, the structure being chosen according to the control objectives and the particular system in question. For example, when the time delay is large, the order of the closed loop can be kept low by employing the forward path PIP controller designed according to Theorem 1 .

It should be noted that the present paper has not addressed the question of a non-constant time delay. The results obtained in Section 7.2 suggest that the SP-PIP
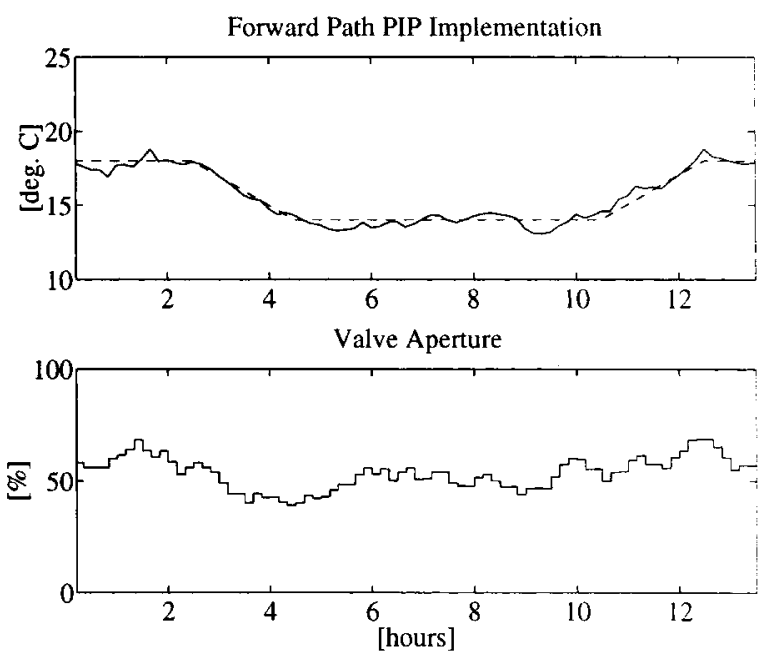

Fig. 8 Greenhouse temperature results for 14 December 1993 (left) and 13 December 1993: comparison of standard feedback (left) and forward path (right) PIP control at $10 \mathrm{~min}$ sampling. [Forward path response reprinted from reference (3) with kind permission from Elsevier Science Limited, The Boulevard, Langford Lane, Kidlington OX5 1GB, UK] 
controller can handle changes in the time delay, but further research is required to evaluate its sensitivity in this regard. In practice, it seems likely that significant temporal changes to the time delay will require some form of adaptive adjustment.

In demonstrating its ability to mimic exactly the SP approach, within an NMSS state space setting, the present paper has illustrated the power and flexibility of the PIP controller, which can be considered as the natural, model-based, successor to the classical PI and PID controllers. In other words, the results presented in this paper, together with those available in previous publications, demonstrate that the PIP approach to control system design provides one of the simplest yet, at the same time, most powerful unified approaches to general digital control system design.

\section{ACKNOWLEDGEMENT}

The research described in this paper has been funded by the Engineering and Physical Sciences Research Council grant number GR/J10136.

\section{REFERENCES}

1 Smith, O. J. M. Closer control of loops with dead time. Chem. Engng Prog., 1957, 53, 217-219.

2 Young, P. C., Behzadi, M. A., Wang, C. L. and Chotai, A. Direct digital and adaptive control by input-output, state variable feedback pole assignment. Int. J. Control, 1987, 46, 1867-1881.
3 Young, P. C., Lees, M., Chotai, A., Tych, W. and Chalabi, Z. S. Modelling and PIP control of a glasshouse microclimate. Control Engng Practice, 1994, 2(4), 591-604.

4 Taylor, C. J., Young, P. C. and Chotai, A. On the relationship between GPC and PIP control. In Advances in ModelBased Predictive Control (Ed. D. W. Clarke), 1994, pp. 53-68 (Oxford University Press, Oxford).

5 Tych, W., Young, P. C. and Chotai, A. TDC: computer aided true digital control of multivariable delta operator systems. In Proceedings of 13th IFAC World Congress, 1996, paper 5c-01 5 (Elsevier Science, Oxford).

6 Taylor, C. J., Young, P. C., Chotai, A., Tych, W. and Lees, M. J. The importance of structure in PIP control design. In IEE Conference Publication 427 of Control 96, 1996, Vol. 2, pp. 1196-1201.

7 Tsypkin, Y. A. Z. and Holmberg, U. Robust stochastic control using the internal model principle and internal model control. Int. J. Control, 1995, 61(4), 809-822.

8 Lees, M. J., Taylor, J., Young, P. C., Chotai, A. and Chalabi, Z. S. Design and implementation of a proportional-integral-plus (PIP) control system for temperature, humidity and carbon dioxide in a glasshouse. Acta Horticulturae, 1996, 406, 115-123.

9 Chotai, A. and Young, P. C. Pole-placement design for time delay systems using a generalised, discrete-time Smith predictor. In IEE Conference Publication 285 of Control 88, 1988, pp. 218-223.

10 Young, P. C. The instrumental variable method: a practical approach to identification and system parameter estimation. In Identification and System Parameter Estimation (Eds H. A. Barker and P. C. Young), 1985, pp. 1-26 (Pergamon, Oxford).

11 Wang, C.-L. and Young, P. C. Direct digital control by input-output, state variable feedback: theoretical background. Int. J. Control, 1988, 47, 97-109. 


\section{APPENDIX}

\section{The $\Sigma$ matrix and proof of Theorem 1}

The $\boldsymbol{\Sigma}_{\text {PIP }}$ matrix which appears in equation (15) of the main text has the following form:

$$
\boldsymbol{\Sigma}_{\text {PIP }}=\left[\boldsymbol{\Sigma}_{\mathrm{f}}\left|\boldsymbol{\Sigma}_{\mathrm{g}}\right| \boldsymbol{\Sigma}_{\mathrm{k}}\right]
$$

where

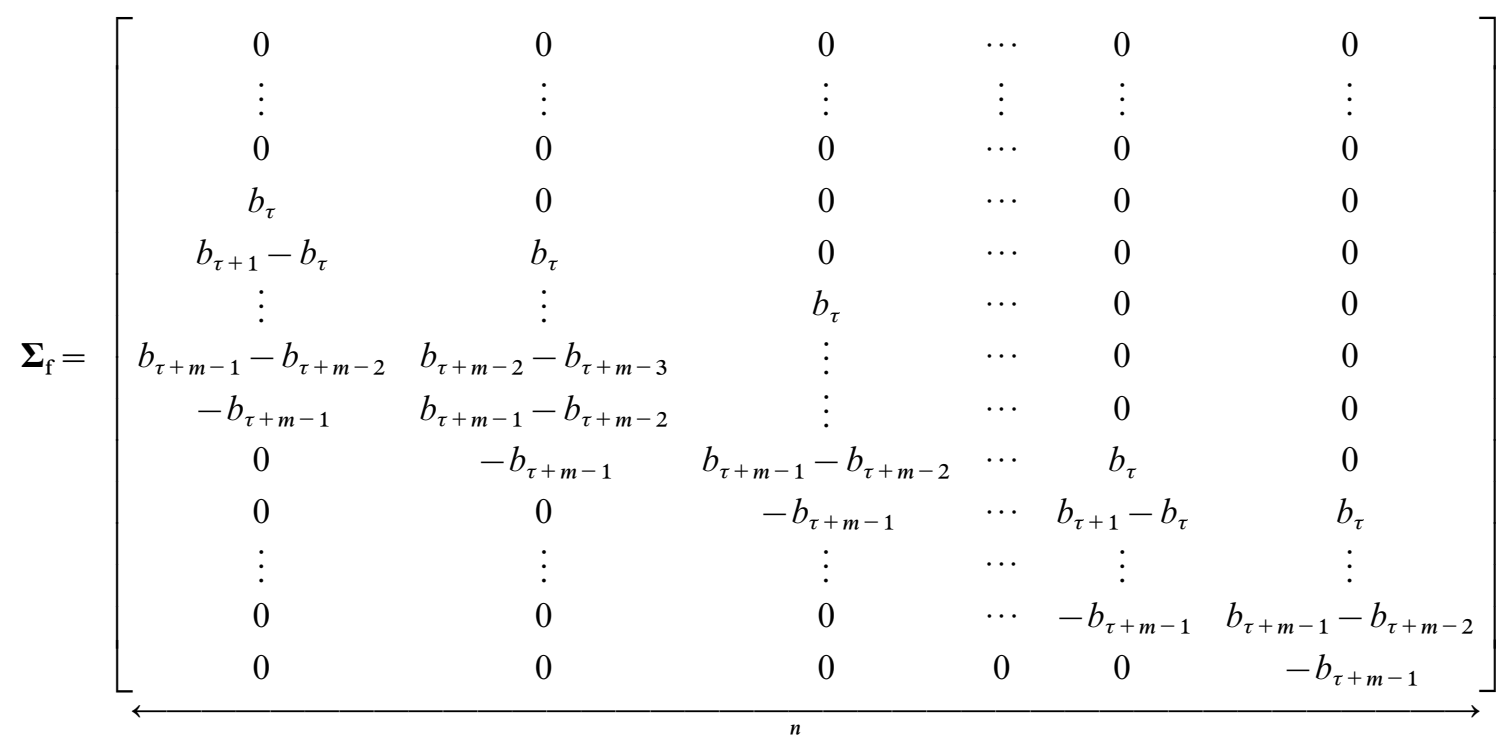$$
\boldsymbol{\Sigma}_{\mathrm{g}}=\left[\begin{array}{ccccc}
1 & 0 & \cdots & 0 & 0 \\
a_{1}-1 & 1 & \ldots & 0 & 0 \\
a_{2}-a_{1} & a_{1}-1 & \ldots & 0 & 0 \\
\vdots & \vdots & \vdots & \vdots & \vdots \\
\vdots & \vdots & \vdots & a_{1}-1 & 1 \\
\vdots & \vdots & \vdots & a_{2}-a_{1} & a_{1}-1 \\
-a_{n} & a_{n}-a_{n-1} & \vdots & \vdots & \vdots \\
0 & -a_{n} & \vdots & \vdots & \vdots \\
\vdots & \vdots & \vdots & -a_{n} & a_{n}-a_{n-1} \\
0 & 0 & 0 & 0 & -a_{n}
\end{array}\right]
$$

\section{Proof of Theorem 1}

The characteristic polynomial of the SP-PIP system is of the order $n+m$, compared with $n+m+\tau-1$ in the standard PIP case. From equations (10) and (14), and with the conditions of Theorem 1, the following equality is obtained:

$$
\begin{aligned}
\nabla\left[G\left(z^{-1}\right) A\left(z^{-1}\right)+\right. & \left.F\left(z^{-1}\right) B\left(z^{-1}\right) z^{-\tau+1}\right] \\
& +k_{\mathrm{I}} B\left(z^{-1}\right) z^{-\tau+1} \\
& =\nabla\left[G_{\mathrm{S}}\left(z^{-1}\right) A\left(z^{-1}\right)+F_{\mathrm{S}}\left(z^{-1}\right) B\left(z^{-1}\right)\right]+k_{\mathrm{S}} B\left(z^{-1}\right)
\end{aligned}
$$

$$
\boldsymbol{\Sigma}_{\mathbf{k}}=\left[\begin{array}{c}
0 \\
\vdots \\
0 \\
b_{\tau} \\
b_{\tau+1} \\
\vdots \\
b_{\tau+m-1} \\
0 \\
0 \\
\vdots \\
0
\end{array}\right]
$$

Note that the first $\tau-1$ rows of $\boldsymbol{\Sigma}_{\mathrm{f}}$ and $\boldsymbol{\Sigma}_{\mathrm{k}}$ are zero.

so that both closed-loop systems have exactly the same denominator dynamics. Moreover, since both systems have a steady state gain of unity, by virtue of the inherent integral action, then $k_{\mathrm{S}}$ must always equal $k_{\mathrm{I}}$ and so the closed-loop numerators are also identical. From the standard PIP pole assignment algorithm, it is well known (2) that

$$
\boldsymbol{\Sigma}_{\mathrm{PIP}} \boldsymbol{k}_{\mathrm{PIP}}=\boldsymbol{d}_{\mathrm{PIP}}-\boldsymbol{p}_{\mathrm{PIP}}
$$

where $\boldsymbol{d}_{\text {PIP }}$ and $\boldsymbol{p}_{\text {PIP }}$ are the vectors of coefficients of the desired closed-loop characteristic polynomial of the standard PIP system and the open-loop characteristic polynomial of the NMSS model (2) respectively, i.e. 


$$
\begin{aligned}
& \boldsymbol{d}_{\mathrm{PIP}} \\
& =\left[\begin{array}{llllllll}
d_{1} & d_{2} & \cdots & d_{n+m-1} & d_{n+m} & \cdots & d_{n+m+\tau-2} & d_{n+m+\tau-1}
\end{array}\right]^{\mathrm{T}} \\
& p_{\text {PIP }} \\
& =\left[\begin{array}{lllllllll}
a_{1}-1 & a_{2}-a_{1} & \cdots & a_{n}-a_{n-1}-a_{n} & 0 & \cdots & 0 & \cdots & 0
\end{array}\right]^{\mathrm{T}}
\end{aligned}
$$

The equivalent result for the SP-PIP case is

$$
\boldsymbol{\Sigma}_{\mathrm{SP}} \cdot \boldsymbol{k}_{\mathrm{SP}}=\boldsymbol{d}_{\mathrm{SP}}-\boldsymbol{p}_{\mathrm{SP}}
$$

where

$$
\begin{aligned}
& \boldsymbol{d}_{\mathrm{SP}}=\left[\begin{array}{lllll}
d_{1}^{\prime} & d_{2}^{\prime} & \cdots & d_{n+m-1}^{\prime} & d_{n+m}^{\prime}
\end{array}\right]^{\mathrm{T}} \\
& \boldsymbol{p}_{\mathrm{SP}}=\left[\begin{array}{lllllll}
a_{1}-1 & a_{2}-a_{1} & \cdots & a_{n}-a_{n-1}-a_{n} & 0 & \cdots & 0
\end{array}\right]^{\mathrm{T}}
\end{aligned}
$$

From conditions (1) and (2) of Theorem 1,

$$
\begin{aligned}
& \quad d_{1}=d_{1}^{\prime}, \quad d_{2}=d_{2}^{\prime}, \ldots, d_{n+m-1}=d_{n+m-1}^{\prime}, \\
& \quad d_{n+m}=d_{n+m}^{\prime} \\
& \text { and } \\
& \qquad d_{n+m+1}=d_{n+m+2}=\cdots=d_{n+m+\tau-2}=d_{n+m+\tau-1}=0
\end{aligned}
$$

If $\boldsymbol{\Sigma}_{\mathrm{SP}}$ and $\boldsymbol{k}_{\mathrm{SP}}$ are padded with an appropriate number of zeros, in order to ensure that the matrices and vectors are all of the same order, then

$$
\boldsymbol{\Sigma}_{\mathrm{PIP}} \cdot \boldsymbol{k}_{\mathrm{PIP}}=\boldsymbol{\Sigma}_{\mathrm{SP}} \cdot \boldsymbol{k}_{\mathrm{SP}}
$$

Note that the $\boldsymbol{\Sigma}_{\text {PIP }}$ matrix is always invertible if the system satisfies the NMSS controllability conditions, i.e. pole assignability conditions (11). Hence,

$$
\boldsymbol{k}_{\mathrm{PIP}}=\boldsymbol{\Sigma}_{\mathrm{PIP}}^{-1} \cdot \boldsymbol{\Sigma}_{\mathrm{SP}} \cdot \boldsymbol{k}_{\mathrm{SP}}
$$

